Revue d'histoire de l'Amérique française

ZRS REVUE D.HISTOIRE DE L'AMÉRIQUE FRANÇAISE

\title{
Gens de couleur libres et colons de Saint-Domingue devant la Constituante (1789 - mars 1790)
}

\section{Gabriel Debien}

Volume 4, numéro 2, septembre 1950

URI : https://id.erudit.org/iderudit/801635ar

DOI : https://doi.org/10.7202/801635ar

Aller au sommaire du numéro

Éditeur(s)

Institut d'histoire de l'Amérique française

ISSN

0035-2357 (imprimé)

1492-1383 (numérique)

Découvrir la revue

Citer cet article

Debien, G. (1950). Gens de couleur libres et colons de Saint-Domingue devant la Constituante (1789 - mars 1790). Revue d'histoire de l'Amérique française, 4(2),

211-232. https://doi.org/10.7202/801635ar d'utilisation que vous pouvez consulter en ligne. 


\section{GENS DE COULEUR LIBRES ET COLONS DE SAINT-DOMINGUE DEVANT LA CONSTITUANTE}

(1789 - mars 1790)

Dejoly nous dit dans ses Mémoires ${ }^{1}$ qu'il fut à partir d'octobre 1789 l'avocat des gens de couleur. "Tandis qu'une société philantropique s'occupait exclusivement de la cause des noirs, je crus qu'il était possible de hâter l'heureux effet de ses travaux et de servir peutêtre plus utilement l'humanité en fixant l'attention du législateur sur une classe d'hommes presque inconnus en France, très nombreuse et infiniment utile aux colonies.

Plusieurs d'entre eux me furent adressés..."

C'est à la fois trop et pas assez dire, et la simplification des faits qui veut sans doute ici, après le 10 août, être prudence et discrétion autour des noms de Brissot, de J. Raimond et d'Ogé, risque de fausser l'histoire. Dejoly grandit légèrement son rôle.

A dire le vrai et le tout, l'affaire des gens de couleur ne commençait pas en octobre 1789 comme Dejoly le laisse entendre. Elle remontait à 1783 et le rôle qu'y tint notre avocat n'est qu'un des épisodes de cette lutte fort longue et assez confuse des gens de couleur libres pour obtenir l'égalité politique avec les blancs. Cette histoire, dont les décrets de mars 1790, du 15 mai et 24 septembre 1791, les concordats coloniaux et la "loi" du 4 avril 1792 sont les pauses principales, devait être l'origine d'une guerre de couleur. Elle explique pour une part le succès de l'insurrection des esclaves et l'appel à l'occupation anglaise.

1. Publiés par J. Godechot, dans les Annales historiques de la Révolution française, octobre-décembre 1946, p. 313. 
Bref, c'est la plus importante des causes de la perte de Saint-Domingue, l'Algérie de notre ancien régime.

Dejoly résume trop les faits en assurant que les Amis des Noirs s'occupaient exclusivement de la cause des noirs. Sans doute n'était-il pas dans leur programme de ménager l'admission des sang-mêlés aux droits des colons. Du moins c'était dans leur esprit, dans celui de Brissot leur fondateur ${ }^{2}$. Evidemment le premier but était l'abolition de la traite. Mais la société crut très vite que soutenir les demandes des libres était un bon moyen de parvenir à la liberté générale. Du rôle de Brissot et de sa société diminué ainsi, Dejoly gonfle d'autant le sien.

"Plusieurs d'entre eux me furent adressés..." Nous sommes au carrefour. Dejoly avoue n'avoir eu aucune initiative. Il faut le croire. Ceux qu'on lui adressa sont Julien Raimond, Vincent Ogé et Fleury et celui qui a dâ les lui amener est Brissot. Si l'on admet que Dejoly était membre des $A m i s$ des Noirs ${ }^{3}$, si l'on observe que Raimond avait d'abord cherché l'appui de cette société, le rapprochement s'éclaire. Brissot ne cite point Dejoly dans ses Mémoires ni Dejoly le nom de Brissot dans les siens. Mais ce silence ne contredit pas l'entente. Raimond et Ogé auront prié Brissot d'agir en leur nom, au grand jour, et Brissot écrivit en faveur des libres. Mais il avait les séances du Comité des Recherches à la Commune de Paris, ses polémiques particulières, son Patriote français à rédiger. Tout en continuant de combattre pour les gens de couleur et de les épauler avec les Amis des Noirs, il demande à Dejoly d'être leur guide, leur plume officielle. Dejoly était lui aussi membre de la Commune, de plus lieutenant de maire, actif, bien pourvu d'amis et d'ambition. Il pourrait se faire agréer par l'Assemblée Constituante comme député régulier des gens de couleur. Les tâches ainsi départagées, les conditions générales de l'offensive paraissaient plus propices, la solution proposée à l'opinion, aux pouvoirs publics, à l'Assemblee, plus claire. Le problème plus simple.

2. Berijamin Frossard a la Convention Nationale sur l'abolition de la traite des nègres (Paris, décembre 1792), in-8.

3. M. Godechot nous l'assure, mais sur aucune liste des membres on ne voit le nom de Dejoly. Pas davantage d'ailleurs celui de l'abbé Grégoire. Dejoly était certainement du nombre des abolitionnistes. 


\section{I}

\section{LA QUESTION DES LIBRES A SAINT-DOMINGUE}

Elle était très ancienne. Les libres, dont les mulâtres formaient la majorité, ${ }^{4}$ avaient reçu par l'article 59 de l'édit de 1685 - qu'on veut appeler le Code Noir - les mêmes droits, privilèges et immunités que les blancs. C'était net. Ce n'était nullement observé, car si à la fin du XVII ${ }^{\bullet}$ siècle la lettre de la loi était pour eux, les mœurs déjà étaient contre. La législation préparée par les soins de Colbert était en retard sur la situation vraie. Elle ne put rien redresser. Les libres étaient franchement à part, mais comme ils étaient peu nombreux, on laissait courir une certaine tolérance: les mariages étaient fréquents entre blancs et libres, et quelques paroisses permettaient le vote des sangmêlés. Puis peu à peu, sans abolir l'édit de 1685 , la règlementation coloniale se tint à l'unisson des usages et des préjugés ${ }^{5}$. Elle mit une barrière infranchissable entre la condition des libres et celle des blancs. Elle les regarda comme des alliés naturels des esclaves, "comme gens de rien, capables tout au plus de servir dans la maréchaussce ou dans les milices". Le Conseil du Cap (1758) leur interdit de porter épée ou sabre, à moins qu'ils ne fussent militaires et en service. En 1762, ce sera les armes à feu et l'achat des munitions qui leur sera défendu. Pour mille choses il leur faut une permission spéciale, pour venir en France par exemple. Un blanc ne peut même pas y conduire sa fille de couleur sans un passeport du gouverneur. Il n'est pas question d'accéder aux charges judiciaires ${ }^{6}$, à des grades, à la noblesse, ou plus simplement d'exercer une profession libérale de médecin, d'apothicaire, voire de chirurgien. Toutes ces incapacités s'étendaient aux alliés blancs des gens de couleur. En 1770, le comte de la Barre, ancien capitaine au régiment de Chamborant et capitaine dans la Légion de

4. Il importe de bien s'entendre sur le sens des mots gens de couleur et mulatres. Il convient de les prendre dans le sens qu'on leur donnait aux îles. Un mulatre est fils d'un blanc et d'une négresse - ou d'un noir et d'une blanche. Un quarteron est issu d'un blanc et d'une mulâtresse. Mulâtres et quarterons sont dits gens de couleur.

5. L.C. Wroth et G.-L. Annan, Acts of French Royal Administration concerning... West Indies... prior to 1791 (New York, Public Library, 1930), in-8.

6. Il n'y avait pas d'offices à Saint-Domingue. 
Saint-Domingue, se vit, sur une plainte anonyme, retirer son commandement pour avoir épousé à Bordeaux l'arrière-petite-fille d'une sangmêlée. Une enquête judiciaire avait pourtant prouvé l'année précédente que madame de la Barre était d'ascendance caraïbe, ce qui ne faisait pas déroger ${ }^{7}$. Le moindre soupçon de sáng noir, une dénonciation malveillante suffisent pour qu'un fonctionnaire soit l'objet d'une enquête. Morts, les gens de couleur restent aussi souvent à part, dans un coin des cimetières ${ }^{8}$.

L'administration centrale eût dans plus d'un cas abaissé cette barrière, mais ses agents étaient, par leurs alliances et les liens de leur fortune, fort engagés dans ce monde colonial. Mais aussi à ses yeux, qui voulaient voir loin, il y avait la raison d'Etat. La justice ne passait qu'après. Une raison de politique générale maintenait ces rangs stricts entre les hommes: il fallait assurer la conservation de la colonie à la France. On croyait qu'accorder la liberté aux libres qui se multipliaient, préparait directement la sécession ${ }^{9}$. Sans interdire à la colonie les mariages entre blancs et libres, les ministres insinuaient aux administrateurs généraux de les décourager, de les déconsidérer, et toute administration a ses trésors de mauvais vouloir. En 1778 ces mariages furent aussi interdits en France, provisoirement ${ }^{10}$.

Cependant après 1780 et la guerre d'Amérique, où les gens de couleur avaient combattu pour la première fois en corps spéciaux hors des Antilles, un mouvement s'était dessiné en faveur des libres, barré d'ailleurs par un contre-courant. Ils prenaient de l'importance. D'abord par leur nombre: ils étaient une vingtaine de mille, à peine moins que les blancs. Puis par leur influence: lors des troubles du sud en 1769 ils s'étaient rapprochés des blancs les plus turbulents, avaient brusque-

7. Archives Nationales, Colonies E/236, dossier La Barre de Laage; E/285, dossier Lévêqué. (Nous désignerons désormais les Archives Nationales par l'abréviation $\mathrm{AN}$ ).

8. C'est ce qu'on répète après Lebeau, De la condition des gens de couleur libres sous l'Ancien régime (Poitiers, 1903), mais qui parâ̂t faux.

9. "Si par le moyen de ces alliances, les Blancs finissaient par s'entendre avec les libres, la colonie pourrait se soustraire facilement a l'autorité du roi". Instructions au prince de Rohan, gouverneur, 1766. - Rd. P. Cabon, Histoire d'Hä̈ti (4 vol. s.d. Port-au-Prince), in-8, II: 306.

10. S.T. Mc Cloy, "Negroes and Mulattoes in Eighteenth Century France", Journal Negro History, XXX (1945): 276-292. 
ment montré le rôle qu'ils pouvaient tenir en période de "remuements". Ils étaient une force qui grandissait. Dans les quartiers neufs ils avaient toujours fait figure de pionniers à côté des petits colons blanes ${ }^{11}$. Mais jusqu'en 1760 le gain des cultures dans les mornes avait été peu important. L'expansion du caféier ouvrit des chances nouvelles de fortune rapide, porta mille gens de couleur vers les hauteurs. Là où il n'y avait jusqu'en 1765 que des places à vivres et à bétail se multiplièrent les petites plantations. Toutes, certes, n'étaient pas propriétés des libres. Mais si, jamais, ils ne possédèrent le quart des terres productives de la colonie, leur part dans les mornes était considérable. Beaucoup vivaient là, indépendants, en famille, aidés seulement de quelques esclaves. Instinctivement ils se rapprochaient les uns des autres, fajsaient masse, isolaient dans ces cantons inhospitaliers les blancs qui s'y étaient fixés.

Dans les plaines elles-mêmes ils gagnaient. Non comme propriétaires de sucreries, comme économes plutôt, ou comme gérants de biens d'absents, de caféières moyennes et d'indigoteries. Ainsi dans le Sud et dans l'Ouest, à Léogane, à l'Arcahaye. Par simple glissement d'autorité, l'habitude se prenait qu'un blanc, père d'enfants de couleur élevés en France, leur confiât l'administration de ses biens lors de son retour au pays natal. On voit la portée de l'évolution. On voit aussi que fatalement, à côté d'une classe de mulâtres incessamment renouvelée par les unions familièrement libres et tenue pour inférieure parce que de naissance irrégulière, il se constituait un fonds de familles de couleur, quarteronnes, issues de mariages légitimes, d'excellentes mœurs, fières, on pense, de leur sang blanc, sérieuses, stables, prospères, "ce qu'il y a de meilleur dans la colonie" était allé jusqu'à dire le gouverneur de Fayet ${ }^{12}$ un soir de découragement ou de sincérité. Avec eux il allait falloir compter. Il avait bien fallu reconnaître déjà le droit d'hériter de leur père blanc à des enfants de couleur nés hors mariage. Les sentiments, et doucement, très doucement, les mœurs faisaient un accroc au préjugé, qui restait avant tout un réflexe de défense politique.

11. Surtout à Gros-Morne, à Aquin, etc. Cf., G.-A. Chevalier, "Etude sur la colonisation française en Haitti. Origines et développement des propriétés Collette", Revue d'histoire et géographie d'Haïti (1938 et 1939).

12. Cabon, op. cit., I: 145. 
Mais la principale force des libres était ailleurs. Les blancs ne venaient à Saint-Domingue que pour faire fortune au plus vite. Ils comptaient les années de leur exil, les mois, les jours. Ils ne faisaient que les projets qui les ramèneraient riches dans la métropole. SaintDomingue n'etait pour un grand nombre qu'un lieu de passage, tout au plus une demi-patrie. Les gens de couleur, eux, n'avaient pas d'autre pays. Ils y étaient nés. Ce n'était pas une colonie pour eux, et leur espérance lointaine était de faire leur, entièrement leur, cette terre où ils trouvaient naturel de mourir. Ils voulaient qu'on leur reconnat en droit la place qu'ils tenaient en fait, et d'abord l'égalité politique. Ils étaient prêts à la demander, cette place, même à la disputer et à se l'approprier. Telle était la question.

Elle se pose plus aigrement après 1770 parce que l'esprit colon devient plus fort. Point chez les plus vieux colons avec lesquels, en fait, les libres s'entendaient assez bien. Les relations entre ces blancs et les mulâtres étaient remarquables par l'absence de violentes rancœurs de race. Elles avaient encore quelques traits de la société du $\mathrm{XVII}^{\circ}$ siècle colonial. Mais après la paix de Paris une nuée de jeunes gens de tous les milieux, pauvres le plus souvent, ambitieux et âpres, est arrivée à la colonie ${ }^{13}$. Les uns se mettent aux défrichements, les autres à leur métier: charpentiers, tonneliers, etc.; d'autres cherchent une place de raffineur, de teneur de livres, d'économe. Ils n'en trouvent qu'avec peine. Ils se heurtent partout à la concurrence des libres qui occupent ou leur disputent ces places, ces métiers ou les terres bonnes à prendre. Ils deviennent leurs adversaires envieux. Et pour maintenir, sans l'autorité de la richesse ou d'un nom qui vous porte, cette force factice qui veut assurer leur suprématie de blancs, ils se font rogues et distants. Des relations d'homme à homme, la familiarité ancienne a disparu, reste du paternalisme, ce confort d'autrefois.

C'est le commencement de la lutte.

13. J. Raimond le note très judicieusement dans ses Observations sur l'origine et les progrès du préjugé des colons blancs contre les hommes de couleur... (Paris, 1791), in-8, 46 pages. Voir aussi $G$. Debien, "A Saint-Dominingue avec deux jeunes économes de plantation", Revue d'histoire et géographie d'Haĩti (juillet 1945). 
LES DEMANDES DE J. RAIMOND (1783-1788)

Ce furent d'abord de pacifiques prières dont un quarteron d'Aquin, dans le Sud, eut toute l'initiative, apparemment $t^{14}$. Cet homme est Julien Raimond. Son père et son grand-père étaient blancs et riches. Dans cette grande paroisse de montagne, aux moyennes et petites plantations, les libres étaient nombreux et unis. Les quarterons y faisaient figure de chefs, et Raimond parmi eux, de conseiller écouté. C'est qu'il avait combattu pour les insurgents d'Amérique, et assisté au siège de Savannah. Au retour, il avait compris la situation morale nouvelle des gens de couleur, soldats de la liberté. Il s'appliqua à en tirer parti. Il s'agissait de grouper les libres, de faire parler d'eux en Europe, de les faire prendre en considération par l'opinion éclairée, par les amis, nombreux, de l'abbé Raynal, et par les amis de ses amis. Il savait l'œuvre longue, mais il avait la redoutable patience, l'entêtement qui fait la force des doux. On le traitera - bien plus tard de pusillanime, mais c'est qu'il ne sera jamais brusque et se refusera aux moyens brutaux. Il n'est pas que des révolutionnaires violents.

Il se met à la tâche qu'il s'est donnée dès son retour à SaintDomingue. La colonie offrait en 1783 un vaisseau au roi. Les libres d'Aquin voulurent contribuer à la souscription. Raimond se fit charger par les administrateurs de recevoir les versements. Il recueillit ainsi 9.410 livres. La belle occasion! "Il assembla chez lui... toutes les personnes de couleur de son quartier, qui, étant réunies, le chargèrent d'une commune voix d'adresser à MM. les administrateurs un mémoire

14. Julien Raimond est né à Bainet, province du sud de Saint-Domingue, le 16 octobre 1744. (Renseignement dû à l'obligeance de M. Luc Nemours). Sa mère, mulâtresse, était légitimement mariée. La fortune des Raimond se composait au moins d'une plantation à Aquin (Moreau de Saint-Méry, Description de Saint-Domingue (2 vol., Philadelphie, 1796-1797), I: 228) et d'une place à Plymouth. Nous n'avons pu recueillir aux Archives de la Charente aucun renseignement sur sa famille et ses biens en Angoumois. C'est dans les fonds notariaux des Archives du ministère des Colonies qu'il conviendrait de chercher. M. Luc Nemours, un Haïtien de Paris, prépare une étude sur la famille de Raimond en France.

Après avoir fait partie de la 3 ème Commission civile envoyée à Saint-Domingue en 1796, Raimond mourut au Cap en vendémiaire an $X$, après cinq jours de maladie, peut-être de la fièvre jaune (Papiers Gérard: renseignement communiqué par Mlle Bl. Maurel). Raimond était alors administrateur général des domaines natiơnaux au Cap. L'étưde de M. M. Cook, "Julien Raimond", Jour. Negro History (avril 1941) n'apprend rien sừ l'origine, les biens ni les démarches de J. Raimond avant 1791. 
des plaintes et réclamations contre le préjuge"'15. C'était une première action commune. Ce mémoire doit être celui que Raimond présenta en 1784 à Bellecombe et à Bongars, le gouverneur et l'intendant, et où il "concluait à demander au gouvernement de mettre des bornes et une fin à la tache qui semble se perpétuer trop longtemps et trop loin"16.

La réponse de Port-au-Prince fut encourageante. Elle montra la marche à suivre. Elle conseillait de porter la requête hors de la colonie, sous les yeux du roi et du ministre. Raimond parlera toujours avec reconnaissance de ce premier accueil et de cette première protection qui seront discrètement reprochés à Bellecombe par la Chambre d'Agriculture du Cap dans son compte rendu critique de l'administration de ce gouverneur ${ }^{17}$.

Raimond passait en France la même année car sa femme avait des intérêts près de Mauzé sur les confins du Poitou et de l'Aunis. Les plus fortunés des libres d'Aquin lui confièrent la mission de porter le mémoire "aux pieds du roi". C'étaient les deux frères Delaunay, les trois Boisrond, Leclerc, Lalanne, Labadie, Lausengues, Maubois, Casamajor, la veuve Montbrun et les deux frères de Raimond, Georges et Pierre ${ }^{18}$. Avant de s'embarquer il fit le tour des quartiers du Sud et de l'Ouest, et recueillit aux Cayes les approbations de Boury, d'Hervieux, d'Hérard, de Marlot, de Ry, à Port-au-Prince, celles de Compaing, Harang, Vimère, Cameau et des Chanlatte, à SaintMarc, celles des trois Pinchinat et d'Abadie. La même réception l'accueillit au Cap. Mais nulle part ne lui fut donnée de procuration en règle, soit pour ne pas faire naître "des soupçons désavantageux" et ne pas être accusé d'assemblées illicites ${ }^{19}$, ou parce que Raimond négligea d'en demander. La mission fut orale. Cela permettra un jour

15. A Maistre du Chambon, "Acte notarié relatif aux doléances des gens de couleur de Saint-Domingue" (29 juillet 1789). Extrait des Mémoires Société archéologique (Charente, 1931), 8.

16. Ibid., 7. Dans la correspondance générale des administrateurs de SaintDomingue avec le ministre au temps de Bellecombe (AN, Colonies, C 9A, 152-156) nous n'avons pas retrouvé ce mémoire ni de lettres à son sujet. Peut-être faut-il chercher aussi dans $C 9 \mathrm{~B}$ et dans la série $\mathrm{B}$.

17. AN, Colonies, F 3, 126, Mémoire du 2 février 1786.

18. A. Maistre du Chambon, 8 et 9.

19. Est-ce ces réninions de libres au passage de Raimond qui provoquèrent l'ordonnance du 11 mars 1785 interdisant aux gens de couleur de s'assembler pour leurs noces, festins et danses sans l'autorisation du Commandant du quartier? 
aux colons de dire à Raimond, selon le besoin, qu'il est à la tête d'une conspiration ou qu'il n'est qu'un rêveur isolé dont l'influence ne dépasse pas les limites de sa paroisse ou la portée de sa famille.

Voici donc Raimond en France. Il est à Paris à la fin de 1785 avec M. de Bellecombe rentré de son gouvernement. Bellecombe reconnut franchement la mission de Raimond et appuya de "toutes ses connaissances" le mémoire remis au maréchal de Castries, ministre ${ }^{20}$. D'ailleurs, vers le même moment, d'autres mémoires venant de la Guyane demandaient aussi au roi l'amélioration du sort des libres. Fitz-Maurice et Lescallier, administrateurs de la Guyane, suggéraient qu'à la quatrième ou cinquième génération les libres fussent assimilés aux blancs ${ }^{21}$. Le ministre adressa la réclamation de Raimond au comte de La Luzern`, le nouveau gouverneur de Saint-Domingue, et à l'intendant BarbeMarbois et les invita le 11 mars 1786 à lui soumettre leurs vues. "Je joins ici deux mémoires qui m'ont été adressés par un homme de couleur qui réclame pour lui et les individus de son espèce protection contre les vexations que leur font éprouver les blancs par l'effet d'un préjugé injuste, ainsi que l'existence dont leurs mœurs, leur aisance, leur honnêteté les rendent susceptibles. L'exposé de leur situation est touchant et il paraît convenable de prendre provisoirement des mesures propres pour mettre cette classe d'hommes à l'abri des vexations dont elle se plaint...22",

Ce n'était pas une manière de noyer le poisson, car dans ses institutions générales au départ de La Luzerne, le 6 décembre précédent, le ministre avait insisté sur les modifications à apporter à la condition des gens de couleur. Il devait consulter les Conseils Supérieurs, les Chambres d'Agriculture, les principaux colons. On lui recommandait “d'étudier s'il n'était pas utile d'apporter quelque tempérament au principe qui excluait à jamais les gens de couleur et leurs descendants de la condition des blancs ${ }^{23}$."

La Luzerne enquêta sérieusement, s'adressa même à l'un des frères de Raimond et à plusieurs habitants du Sud pour confirmation

20. G. de Perceval, Le Comte Laîné (2 vol., Paris, 1929), I: 47, note.

21. Lebeau, op. cit., 6 “Mémoire au ministre" du 15 janvier 1786.

22. AN, Colonies, B 192: 43.

23. Lebeau, op. cit., 7. AN. Colonies F 3, 72, 30 juillet 1786. 
des faits cités dans les mémoires présentés à Versailles"24. Puis, en 1787, il passa du gouvernement de Saint-Domingue au ministère. Il s'était montré compréhensif, on s'attendit à le voir généreux et juste. Mais Raimond commit une erreur: au lieu de rester à Paris auprès des bureaux il alla en Angoumois. C'était rentrer sous terre. Il perdit contact avec ses premiers protecteurs de Versailles. La bonne volonté du ministre s'émoussa en se heurtant aux mœurs et à la mauvaise volonté des colons. La Luzerne n'était pas un homme de combat. Au moment où à Saint-Domingue et à Paris s'ouvrait la question de la représentation des colonies aux prochains Etats généraux, aucune décision n'avait été prise: on en était aux examens, aux considérations.

Quelques planteurs avaient adopté, il est vraj, à l'égard des libres une attitude demi favorable. Ce même bon sens avisé dirigeait la prévoyance des négociants des ports. Mais tous ces "esprits nouveaux" étaient éloignés d'accorder d'emblée, même à l'élite des libres, l'égalité politique. Ils admettaient la nécessité, voire l'urgence de concessions: ainsi le droit d'entrer aux assemblées paroissiales. Pour eux il y avait une question des libres.

A côté, l'immense majorité des colons était farouchement opposée à ce début d'assimilation. Il était évident à leurs yeux que l'octroi des droits civiques aux libres, riches et nés libres, entraínerait vite l'affranchissement des esclaves. Et un demi-continent était derrière eux.

\section{$*^{*} *$ \\ III \\ LA QUESTION DES GENS DE COULEUR DANS LES CAHIERS}

On ne s'attend pas à voir les cahiers généraux des sénéchaussées de France prendre feu et cause pour les gens de couleur de Saint-Domingue. En fait on ne trouve pas d'autre trace de la question des libres qu'une phrase $d u$ cahier du Tiers de la ville de Paris". Il dit: "Les

24. M. Laurent, "Une des premières réclamations des affranchis à SaintDomingue" (1789), dans le Document, (3 mars 1940).

25. Communication de Miss B. Hyslop qui a relevé tous les passages des cahiers généraux de sénéchaussée où il est question de la traite et de l'esclavage. 
États généraux prendront en considération le sort des esclaves noirs, ou hommes de couleur, tant dans les colonies qu'en France."26 Bien que l'expression hommes de couleur fat peu en usage à Saint-Domingue, on ne peut douter de sa provenance. Derrrière le rédacteur du cahier il y a évidemment un ou plusieurs mulâtres libres, et l'influence des Amis des Noirs.

Pourtant il serait surprenant que Raimond ne fût pas parvenu par la voie des $A$ mis des Noirs à glisser ses plaintes à côté des doléances de certaines communautés d'habitants. Les cahiers de paroisse n'ont pas encore été examinés sous ce jour. Il serait nécessaire de commencer par la région d'Angoulême où habitait Raimond et par les environs de Paris où la propagande de Brissot et des Amis des Noirs fut plus efficace. Il n'est pas assuré que cette recherche aboutisse a des précisions: à Angoulême Raimond a pu cacher ses origines de couleur. Autour de Paris l'action de Brissot a pu gauchir les id́es et les demandes de Raimond. Brissot souhaitait l'émancipation des esclaves. Il la préparait, en demandant la suppression immédiate de la traite. Rien de tel chez Raimond, propriétaire d'esclaves, qui réclamait seulement l'égalité des libres avec les blancs. Brissot aura associé ce vœu aux siens, plus généraux et moins immédiatement réalisables.

Mais la question ne pouvait être étrangère aux cahiers des colons de Saint-Domingue. Le plan proposé en 1789 par la colonie pour la formation des Assemblées coloniales, dit expressément à propos du droit électoral: "Sous la dénomination de propriétaires planteurs seront compris les propriétaires de maisons dans les villes ou bourgs et généralement tous ceux qui auront une propriété foncière équivalente à une habitation de... 25... esclaves, bien entendu que dans les planteurs ou propriétaires de maisons ne peuvent être comptés les gens de couleur libres ou leurs alliés en ligne directe"2r. Et l'on rejetait l'idée que le comte de Reynaud avait exprimée dans le Discours préliminaire du plan:

"Les gens de couleur libres, à quelque distance qu'ils soient de leur origine, conservent toujours la tache de l'esclavage et sont déclarés

26. Ch.-L. Chassin, Les élections et les cahiers de Paris en 1789 (4 vol., Paris, 1888-1889), III: 359.

27. Mlle Blanche Maurel, Cahiers de doléances de la colonie de Saint-Domingue pour les Etats généraux de 1789 (Paris, Leroux, 1933), 287. 
incapables de toutes fonctions publiques. Les gentilshommes mêmes, et qui descendent à quelque degré que ce soit d'une femme de couleur, se trouvent soumis à cette loi, dure à la vérité, mais sage et nécessaire; on ne saurait dans un pays où il y a vingt nègres pour un blanc, mettre trop de distance entre les deux espèces; l'administration doit donc être attentive à maintenir cette distance et ce respect. En conséquence de ce principe les nègres et les gens de couleur libres ne votent pas, même dans les assemblées provinciales ordinaires, quelques propriétés qu'ils aient, mais il parait juste qu'ils aient le droit de s'y faire représenter par un procureur blanc"28.

Le cahier de doléances de la Chambre d'Agriculture du Cap était aussi absolu que le Plan, "...les gens de couleur libres, ceux [ des blancs ] qui auront épousé des femmes de couleur, ne seront point admis aux dites assemblées [ de paroisse ]"29. La brièveté rude de la formule apprenait sans détour ce qu'il fallait entendre par vœu et désir des colons.

Cette volonté bien arrêtée d'interdire aux libres l'accès des assemblées de paroisse paraît avoir eu une grande influence sur le programme de Raimond. A voir l'intention précise des colons il comprit où devait frapper l'effort premier: il fallait d'abord demander l'entrée aux assemblées de paroisse. Aux indifférents de France une telle prière paraîtrait modeste, la réforme bénigne. La réalité était tout autre et les gens de Saint-Domingue le savaient bien. Jusque là l'usage excluait les libres de ces assemblées, encore qu'il y eût quelques exceptions. En certains cas il avait paru impossible d'écarter de délibérations d'intérêts communs tels riches propriétaires de couleur. Ces assemblées n'avaient rien d'assemblées politiques. Et la veille des grandes réformes, les faiseurs de plans envisagent ces petites assemblees sous un jour nouveau. Elles étaient des corps chargés de l'administration locale. Elles deviennent les chambres électorales qui choisiront les membres des Assemblées provinciales. Elles seront le fondement de l'édifice législatif. Il devient donc d'une importance décisive d'enlever aux libres tout pouvoir d'y accéder. Dans la plupart des paroisses assurément ils seraient en minorité. Mais dans le Sud, à Aquin par exemple, les admettre au vote, c'est verser toutes les voix sur le même nom, qui sera

28. Ibid., 166.

29. Ibid., 316. 
un nom de personne de couleur; c'est leur abandonner certaines paroisses, leur donner la chance d'être représentés aux assemblées provinciales. Le souci, le raisonnement va loin. Surtout il allait vite. La logique et la crainte des colons fermaient cette porte basse. La logique et l'espoir de Raimond sera de la forcer.

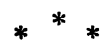

IV

\section{LA PREMIERE IDEEE D'UNE DEPUTATION}

L'agitation des colons pour obtenir des députés aux Etats généraux va venir compliquer le plan de Raimond. A la fin de 1788 , il lui apparut que seule une députation parviendrait à obtenir l'entrée aux assemblées de paroisse. Il faut aux libres une représentation parallèle à celle des blancs. D'ailleurs Raimond ne sembl ' pas avoir songé à ce rôle de député pour lui-même. Mais au lieu d'adresser directement sa requête aux bureaux de Versailles, il préfère que ses compatriotes du Sud et ses amis d'Aquin fassent la démarche. Son frère François, Dabadie, Boisrond et Lamarre adressent cette supplique aux administrateurs généraux, au marquis du Chilleau et à Barbé-Marbois, le 15 mars 1789:

"Nosseigneurs, Notre classe pénétrée du désir de faire connaître au Roy son zèle et son patriotisme aux Etats généraux n'a pas osé jusqu'à présent vous en demander la permission, mais apprenant que la première classe y envoie des députés nous prenons la liberté de vous exposer, Nos Seigneurs, qu'en France les Etats généraux sont composés du Clergé, de la Noblesse et des roturiers, ces derniers connus sous le nom de Tiers-Etat. A Saint-Domingue la colonie est composée de blancs et de gens de couleur. Si les blancs sont admis aux Etats généraux comme étant la première classe, nous osons espérer que nous devons y être aussi admis, comme étant la dernière classe. Nous n'entreprendrons pas de pénétrer les motifs qui portent la première classe à demander l'admission aux Etats généraux; nous désirons seulement faire connaître au Roy à la tête de la Nation assemblée qu'il y a une classe 
de ses sujets éloignés de 2.000 lieues qui l'aime et qui est dans l'avilissement et dans l'opprobre et que le préjugé tient dans l'abrutissement... Si vous nous jugez dignes d'être admis aux Etats généraux nous prendrons la liberté de vous adresser un mémoire pour faire parvenir au Roy à la tête de la Nation assemblée"30.

Les administrateurs faisaient suivre cette lettre au ministre le 20 mai, sans commentaires ${ }^{31}$, et le $1^{\text {er }}$ aout, La Luzerne en accusait réception: "Il ne m'appartient pas de prononcer sur cette réclamation. La colonie ayant actuellement des représentants aux Etats généraux c'est à eux de mettre la demande des gens de couleur sous les yeux de l'Assemblée Nationale"32.

Raimond se tourna alors vers un gentilhomme de l'Angoumois, qu'il connaissait sans doute depuis longtemps. Charles de RohanChabot, comte de Jarnac, maréchal de camp, inspecteur de la $15^{\text {dme }}$ division de cavalerie ${ }^{33}$, "demeurant ordinairement en son hôtel de Paris, rue de Monsieur" ${ }^{34}$ n'avait qu'une médiocre réputation en son comté, qui d'ailleurs ne le voyait guère. Il était hautain, dédaigneux, généralement mal vu de la noblesse, qu'il toisait. Mais il voulait jouer au libéral et il possédait des intérêts à Saint-Domingue. Il est probable qu'il avait songé à la députation lors des élections de mars 1789. Son nom, ses relations à la Cour pouvaient paraître une force. Raimond lui proposa de présenter aux États généraux les demandes des gens de couleur, d'être leur porte-parole et leur protecteur. Et Jarnac accepta cette charge qu'il paraît avoir désirée. Il correspondait avec les libres du Sud:

30. M. Laurent, 168.

31. Port-au-Prince, 20 mai 1789. AN, Colonies, C 9A, 162.

32. M. Laurent, 171.

33. Marie-Charles-Rosalie de Rohan-Chabot (1740-1813), fils de Guy-Auguste, duc de Rohan, vicomte puis comte de Jarnac en 1751, entré au service à 14 ans; maréchal de camp en 1781, lieutenant-général en 1789. Emigré en 1792, fait la campagne en 1794, meurt en Angleterre. De son premier mariage avec Guyonne-Hyacinthe de Pons - propriétaire a Léogane - il n'eut qu'une fille. Son second mariage avec Elisabeth Smith le tenait aussi proche de Saint-Domingue.

34. L'hôtel de Jarnac, 8 rue Monsieur, dû aux plans de Legrand, aurait été construit en 1783 par Léonard Chapelle. Le comte de Jarnac n'en aurait été que le locataire. Il y aurait un autre hôtel de Jarnac, habité sous Louis XV par Charles-Annibal de Rohan-Chabot et situé rue de Bourbon (communication de M. Martin-Civat, $B^{i n}$ et Mém. Societé archéologique et historique Charente, XLVIII, 1946). 
A la Colline d'Aquin, 3 juillet 1789 - "Nous voyons avec douleur, écrit à Raimond son ami Labadie, que depuis six mois que vous êtes en France pour réclamer contre les vexations que nous éprouvons ici de la part des Blancs, vous n'avez pu parvenir à rien obtenir du gouvernement en notre faveur, mais un jour nouveau paraît luire pour notre bonheur. Des Etats généraux assemblés pourront nous entendre si vous réclamez pour nous comme vous nous le faites espérer et ainsi que l'écrit aussi M. le comte de Jarnac à votre frère. Il est heureux pour nous que ce seigneur veuille bien s'intéresser à notre cause.

Vous nous mandez l'un et l'autre qu'il serait nécessaire que nous nous assemblions ici, et que nous nommions quelques commissaires entre nous. Hélas! vous n'ignorez pas que le fatal préjugé nous en empêche. Nous avons même fait quelques démarches à ce sujet, et votre frère et moi, avons écrit en avril dernier ${ }^{35}$ à $\mathrm{M}$. Duchilleau pour lui en demander la permission. Nous avons été sans réponse. Ce silence constant de sa part nous prouve que les Blancs l'ont gagné et nous ne sommes pas à nous apercevoir qu'on nous surveille encore plus qu'auparavant pour nous empêcher de nous assembler, et nous n'osons nous y exposer, surtout ceux qui ont du bien. Nous ne voulons point donner la moindre prise contre nous...

M. Auge é $^{36}$ a déjà écrit à nos Messieurs du Cap, de Saint-Marc ${ }^{37}$. Il nous ont dit avoir déjà reçu une lettre de ces derniers, par laquelle il mande que vous êtes au moment de vous réunir et que vous vous décidez à faire des démarches pour avoir une députation. Il n'est pas douteux que ce serait le vœu général de tous, mais comment vous le faire parvenir... J'ai engagé plusieurs des nôtres à vous écrire. Je ne sais si leurs lettres vous parviendront, vous savez que plus d'une fois on s'est permis de les ouvrir et de les retenir...."

Tout cela nous montre que les rapports de Raimond avec ses compatriotes du Sud n'étaient pas très réguliers et qu'en juillet 1789 la mission dont Raimond était chargé était encore sans confirmation écrite en forme.

35. Allusion aux pétitions adressées en mars.

36. Vraisemblablement Vincent Ogé. Il était fils d'un boucher du Cap et d'une femme de couleur.

37. Peut-être au moment de son embarquement pour la France.

38. M. Laurent, 165. 
Le 29 juillet 1789, à Jarnac, par devant notaire, Raimond constituait le comte de Jarnac son représentant et lui confiait "le patronage de la classe des gens de couleur libres", "avec plein pouvoir pour faire agréer et recevoir aux Etats généraux les remontrances de la dite classe... et... obtenir une loi qui fasse jouir à l'avenir les personnes de couleur à la seconde génération de la légitimité ingénue..." ${ }^{39}$.

Entre Raimond et Jarnac y eut-il quelque différend? Le comte fut-il pris par d'autres soucis? Ne put-il se rendre immédiatement a Paris ? On le voit tout l'été a Jarnac ${ }^{40}$. Se rendit-il vite compte de l'hostilité irréductible des colons qui résidaient en France ? Ou se chargea-t-on de lui faire comprendre que son intervention n'a vait aucune chance de succès, même qu'elle ne pourrait lui valoir que des désagréments? On a le choix entre ces diverses hypothèses. Mais la plus vraisemblable est que plusieurs de ces colons qui commençaient à se concerter durent très précisément aider Jarnac à se persuader de ces difficultés. La persuasion dut être aisée; car Jarnac ne paraît avoir jamais fait partie des Amis des Noirs. A la fin de 1789 il sera inscrit au club Valois ${ }^{41}$. A partir du mois d'aout, Raimond ne parle plus du comte de Jarnac.

D'après une lettre de Baldy, quarteron fixé à Pelissanne, près d'Aix, Raimond lui aurait offert la députation. Cette lettre est du 18 septembre, l'offre de la fin d'aout. Baldy refusa parce que les 6 millions que Raimond avait offerts à l'Assemblée Constituante lui semblaient au-dessus des moyens des gens de couleur. Puis se faire représentant des mulâtres aurait découvert sa couleur, ignorée autour de lui en Provence ${ }^{42}$.

C'est seulement après ce refus que Raimond recourut aux offices de Dejoly.

39. A. Maistre du Chambon, 10. L'acte était passé par devant Guignebert et Deszile.

40. Renseignement communiqué par M. Martin-Civat, bibliothécaire de la ville de Cognac.

41. Challamel, Les clubs contre révolutionnaires (Paris, 1895), 49.

42. M. Laurent, 168. 


\section{PREMIERS CONTACTS DE RAIMOND AVEC LE CLUB MASSIAC}

A leur première réunion, le 20 aout, les planteurs qui devaient peu après fixer leur siège à l'hôtel Massiac apprirent qu'un homme de couleur de Saint-Domingue était chargé de réclamer auprès du ministre en faveur de ses compatriotes. Sans doute cette démarche avait-elle pour but d"'améliorer leur état civil". Duval Sanadon, un des colons, pensait que cette réforme devait faire partie des objets dont l'Assemblée de la Colonie aurait à s'occuper "pour aviser à y faire droit selon le vœu de l'humanité bienfaisante, sans porter cependant préjudice et atteinte aucune aux droits et au régime habituel et nécessaire de la colonie"43.

Le vicomte Alexandre de Lameth propose aux colons présents d'accepter une partie des demandes des gens de couleur afin de les avoir pour alliés au cas où les actes de l'Assemblée Nationale amèneraient des troubles aux Antilles, et notamment une insurrection des esclaves $^{44}$. Quelques esprits prudents partagent ces idées. L'abbé d'Osmond remet à l'assemblée des colons un mémoire qui résume les moyens d'améliorer le sort des libres ${ }^{45}$. L'abbé de Paroy aurait voulu donner aux gens de couleur et aux nègres libres comme "aux gérants, économes, apprentis et ouvriers de toute espèce", le droit d'assister aux séances des comités de paroisse. "Il est bon de leur témoigner une espèce de considération, de les ménager. Le moment d'en avoir besoin est peut-être plus près qu'on ne croie" ${ }^{\prime 46}$. Stanislas Foache, négociant colonial, Barré de Saint-Venant, Fleuriau de Touchelongue, Laborie, un des députés envoyés par la colonie aux Etats généraux, se

43. AN, Dxxx85, dossier 827, pièce 9.

44. La pièce est curieuse. Si curieuse qu'elle paraît douteuse. Elle n'est pas signée (Dxrx85/822/pièce 7) et c'est au moment de l'enquête dirigée en l'an III par GarranCoulon qu'on a écrit en haut du document: Motion de M. le Vte de Lameth, à l'Assemblée du 20 aout 1789. (Cf., Garran-Coulon, Rapport sur les troubles de SaintDomingue (4 vol., Paris, ans V-VII), I: 129; II:5). Alexandre de Lameth, le vicomte, n'avait aucune propriété a Saint-Domingue, mais son frère Charles, le comte.

45. AN, W 15. Procès verbaux des séances du club (24 août-31 déc. 1789), séance du 26 déc. au soir.

46. AN, D×xv85/822 /pièce 17 . 
montrent partisans d'un examen de la cause des libres, au moins des quarterons. D'autres y ajoutent même de la sympathie, comme le chevalier des Landes ${ }^{47}$. On est aux premiers jours du club Massiac.

Pendant ce temps la Constituante a discuté les termes de la Déclaration des droits. Elle vient de voter que "tous les hommes naissent et demeurent libres et égaux en droits". C'est un levier pour la cause des sang-mêlés. Raimond s'en sert aussitôt. Le 25 août, à la séance du soir, le bruit court au club Massiac qu'un mulâtre s'est présenté le matin même à l'Assemblée Nationale pour réclamer l'octroi des droits civiques aux quarterons ${ }^{48}$. Le bruit est faux, mais il montre de quelle surveillance inquiète certains colons entourent les démarches de Raimond. Les mulâtres tiennent déjà des séances, ont un plan d'action. Raimond a l'intention de présenter au plus tôt sa pétition à l'Assemblée. Mais il veut auparavant prendre contact avec le club des colons dont il sait les opinions divisées.

Il s'y fait annoncer le 26 au matin. Dans son esprit ce n'est guère qu'une visite de courtoisie. Tous les détails de l'accueil semblent avoir été préparés. A son entrée "tous les membres restent assis"49. Il exprime au bureau son plaisir de savoir les colons assemblés pour le bien général de la colonie et rappelle le mémoire qu'il avait présenté à Bellecombe et au ministre. On lui demande l'objet de ses réclamations et une copie de ce mémoire. Raimond n'a point ce mémoire ici, et pour une première visite commet la maladresse de citer les Considérations d'Hilliart d'Auberteuil ${ }^{50}$ qui étaient aussi détestées des colons qui lisaient que pouvait l'être l'Histoire philosophique des Deux Indes. Il désirait, comme d'Auberteuil, qu'on fixât un degré de légitimité après lequel les libres seraient tenus pour ingénus. Raimond n'avait pas de pouvoirs à montrer. La réponse du président fut d'un régent: pas de procuration, pas de mémoire, et donc pas d'auditeurs. L'on devait attendre le texte des demandes pour les examiner. Raimond en promit l'envoi et se retira.

47. AN, Colonies, C 9, B 40, 27 juillet 1789.

48. AN, W 15. Procès-verbal du 25 aoút.

49. AN, W 15. Procès-verbal du 26 aout au matin.

50. Considérations sur l'état présent de la colonie française de Saint-Domingue (2 vol., Paris, 1776-1777) supprimées par arrêt du Conseil dès le 17 décembre 1777. En 1780 le ministre suscita une réponse, les Nouvelles considérations sur Saint-Domingue, par P. U. Dubuisson. 
L'accueil avait été poli, mais réticent. On avait cherché à démonter Raimond, à diriger la visite pour qu'elle ne tournât pas en discussion. Elle eut deux conséquences. Elle fit rebondir la question des libres au sein du club et centrer l'attention des "observateurs" du club sur Raimond et ses mouvements. Lui qui n'était pas d'humeur cassante et qui allait tout de suite à l'espérance croyait qu'on trouverait à s'entendre du moment qu'il ne réclamait pas les droits civiques pour tous les gens de couleur mais seulement pour les quarterons de descendance légitime et libres depuis deux générations. Il pouvait penser qu'on ferait en face quelque sacrifice d'amour-propre puisqu'on s'accordait sur le principe que la liberté était une chose et l'égalité une autre, la seconde n'étant pas due à tous ceux qui jouissaient de la première. Raimond semblait reconnaitre, tout comme les colons, que l'égalité était un acte de bienveillance. C'était même leur mot, qui conservait toute distance et leur maîtrise. Il maintenait leur paternalisme en publiant qu'ils n'étaient pas si fermés qu'on le répétait aux idées libérales.

En réalité Raimond devint une des bêtes noires du club. Toutes ses intentions et ses démarches furent désormais déformées avec malveillance. On s'en rend compte dès le 28. A la séance du soir, un colon lut un "projet des mémoires que les mulâtres se proposaient de présenter" à la Constituante. D'après ce texte Raimond aurait eu l'intention, non seulement de demander l'égalité complète de tous les gens de couleur libres avec les blancs, mais l'abolition de la traite, la citoyenneté de tous les enfants nés d'un blanc et d'une négresse et l'affranchissement de tous les nouveau-nés noirs. Cela dépassait singulièrement les demandes de Raimond. Mais rien ne déforme tant qu'un résumé, pour peu qu'on le veuille. Les Amisdes Noirs n'auraientils pas glissé leurs vœux à côté de ceux des mulâtres? N'y aurait-il pas aussi la main du club Massiac? De telles demandes, si abruptement présentées, étaient pour amener les colons hésitants à se rallier à la politique de ceux qui voulaient maintenir fermée la barrière des couleurs. Cependant, que Raimond et les libres qu'il représentait aient offert d'affranchir d'un coup tous leurs esclaves, ne paraît pas avoir été inventé. Nous en avons un écho, peu clair il est vrai, dans la lettre de Baldy du 18 septembre ${ }^{51}$.

51. M. Laurent, 168. 
Au club, Duval Sanadon est l'homme de "l'affaire des gens de couleur". Il apprend aux colons que le mémoire de Raimond va être lu à l'hôtel de ville. Il reste en rapport avec Raimond qui lui a promis, encore, d'envoyer aussi ce texte au club ${ }^{52}$. Les colons s'inquiètent. Ils ont peur que les libres et même les esclaves ne rapportent à la colonie les idées nouvelles. Au début de juillet déjà, des négresses débarquées avec leurs maîtres, leur passeport en règle, avaient été aussitôt mises au dépôt pour être au plus tôt renvoyées à SaintDomingue ${ }^{53}$. Parmi les passagers qui s'embarquent, comment distinguer les pacifiques colons des agents de Brissot et de Raimond? Tout est crainte. Le 20 aout un arrêté du club veut enjoindre à tout colon qui repart de laisser ses domestiques de couleur en France, et la séance du 24 se clôt sur la rédaction d'une lettre à tous les amis de province. C'est une invitation à surveiller leurs gens.

La visite de Raimond au club fait accentuer cette interdiction. Le 27, le plan de convocation des Assemblées coloniales présenté par l'abbé de Paroy propose de défendre à tout noir ou mulâtre arrivant d'Europe de débarquer à Saint-Domingue. Mais les gens de couleur sont seuls l'objet de la circulaire que le club adresse aux villes de commerce, précipitamment ${ }^{54}$. C'est même par cette demande qu'il entre en liaison avec les ports. Le 31 août les colons "examinent les moyens que quelques membres ont employés pour empêcher que les assemblées des mulâtres ne fussent nuisibles aux intérêts de la colonie". Les chambres de commerce de Saint-Malo, du Havre, les Consuls de Nantes, les 3, 5 et 9 septembre ${ }^{55}$ répondent avec toute la bonne volonté possible. Tous s'emploieront à empêcher tout départ de libres "ayant séjournés en France". Les réponses des colons que l'on a pu prévenir individuellement sont plus précises encore. Mais entraver le retour des libres paraît à beaucoup de négociants des ports moins facile qu'aux gens de Paris, et injuste. Ils font des distinctions.

52. AN, W 15. Séance du 28 aout au soir.

53. J.-J. Carles au chevalier de Nonainville, 15 juillet 1789 . Bibl. Saint-Lô, Ms. 154. Et M. Besson, "La police des noirs sous Louis XVI en France", Revue d'Hist. Col. Franc. (1928): 433-446.

54. AN, W 15. Procès-verbal des séances des 26 et 27 aout.

55. A Nantes, Drxv 85/ 822/ 4 à Saint-Malo, id., 823/25; au Havre, W 15. Procès-verbal de la séance du 10 septembre au soir. 
La société de colons qui s'est formée à Bordeaux au premier appel du club promet le 8 septembre qu'elle fera tous ses efforts, mais contre "les gens de couleur non propriétaires", comme si la possession d'un bien était une assurance contre tous mauvais desseins ${ }^{56}$.

Les députés extraordinaires du commerce et des manufactures sont eux-mêmes circonvenus et le ministre imploré. Sans s'arrêter à l'inconséquence, les colons qui ont tant crié contre le despotisme ministériel, sollicitent une circulaire, sans plus, qui d'un mot fermerait les frontières maritimes. Dieu! à quoi servait donc l'autorité! La Luzerne vit les choses de plus haut. Lui aussi avait son intérêt à sauvegarder. Il $\mathrm{y}$ avait les principes, les lois à respecter, la liberté à conserver et dans un moment où l'opinion se posait en surveillante de tous les actes du gouvernement. Le ministre ne put qu'offrir ses exhortations, et bien indirectes, aux commissaires des ports. Il voulait être utile, mais en conservant la face. Il parut neutre aux colons. Certains d'entre eux se souviendront farouchement de cette neutralite ${ }^{57}$.

Officiellement, le club aussi veut paraître neutre, accueillant aux efforts des libres. Vincent Ogé est maintenant en France avec son frère Jacques. Il est beaucoup plus bouillant que Raimond. Il propose aux sang-mêlés de Paris de se rendre en groupe auprès des colons pour s'entendre avec eux après une discussion loyale. Mais il se trouve seul le jour où il doit se présenter au club. Est-il en désaccord avec Raimond? Il obtient de lire devant les colons assemblés un long "plan de régénération pour la colonie" ou plutôt une introduction au plan qu'il soumettrait aux colons s'ils l'y autorisaient, 7 septembre. "Ce quarteron, ajoute le procès-verbal, a laissé son mémoire sur le bureau. Une réponse lui sera faite après délibération". Il n'a donc point lu son mémoire.

Ce mémoire devait être assez bénin de fond, et très respectueux. Le discours de présentation que nous a conservé Madiou ${ }^{58}$ insiste sur le danger d'une insurrection générale des esclaves, mais paraît admettre des époques et des conditions dans les libertés à accorder. Il offrait un terrain d'accord. Mais était-ce pour être écouté plus facilement qu'Ogé ne parle qu'en son nom, comme si douze jours avant,

56. Bordeaux, 8 septembre. Dxxv 85/823/10.

57. W 15, Procès-verbal du 11 septembre.

58. Histoire d'Haïti (3 vol., Port-au-Prince, 1847-1848), III: 29. 
Raimond n'était pas venu devant les mêmes colons plaider"la même cause?

Ce plan en tout cas souffle sur la peur des colons. Le jour même ils écrivent à Bordeaux: ils redoutent que "les démarches des gens de couleur ne produisent une insurrection, ou du moins beaucoup de désordre" ${ }^{59}$.

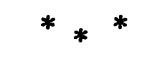

(à suivre)

Gabriel Debien.

Le Caire, (Egypte)

59. AN, W 15, Séance du 7 septembre. 